\title{
Single satellite beam scanning positioning based on Neural Network BP algorithm
}

\author{
Yongwei $\mathrm{Li}^{1, \mathrm{a}}$, Yuwen Wang ${ }^{2, \mathrm{~b}}$, Hanjing $\mathrm{Li}^{2, \mathrm{c}}$, Qiang $\mathrm{Shu}^{2, \mathrm{~d}}$ and Manchuan Zhao ${ }^{2, \mathrm{e}}$ \\ ${ }^{1}$ University of Electronic Science and Technology of China, School of Aeronautics and Astronautics, Chengdu, China; \\ ${ }^{2}$ University of Electronic Science and Technology of China, School of Aeronautics and Astronautics, Chengdu, China;
}

\begin{abstract}
In this paper, the principle of single line positioning based on beam scanning and the neural network algorithm are analysing, and the neural network BP algorithm is applying to the single satellite positioning. At the same time, this paper presents a new algorithm based on electron beam (MEO) for the single scan positioning (Middle Earth orbit). Finally, through theoretical analysis and simulation, it is proving that the neural network BP algorithm of single satellite beam scanning is feasible in fast positioning.
\end{abstract}

\section{INTRODUCTION}

Single-satellite beam scanning positioning technology, that beam information from a satellite, with beam scanning positioning technology to detect the target and the coordinates of the target location solution [1,2]. When the satellite is running near the apogee, the satellite can cover the target area for a long time. The handheld terminal in the target area can receive the beam scanning information of the satellite within the time period covered by the satellite pair area, and the handheld terminal collects enough beam information through the signal processing, positioning operations can calculate their own latitude and longitude coordinates [3].

\footnotetext{
${ }^{\text {a } Y o n g w e i ~ L i: ~ y w l i 0314 @ s i n a . c n ~}$

b Yuwen Wang:83211668@qq.com

${ }^{c}$ Hanjing Li: 448063460@qq.com

${ }^{\mathrm{d}}$ Qiang Shu: 874611955@qq.com

e Manchuan Zhao: zmc zhao@163.com
} 


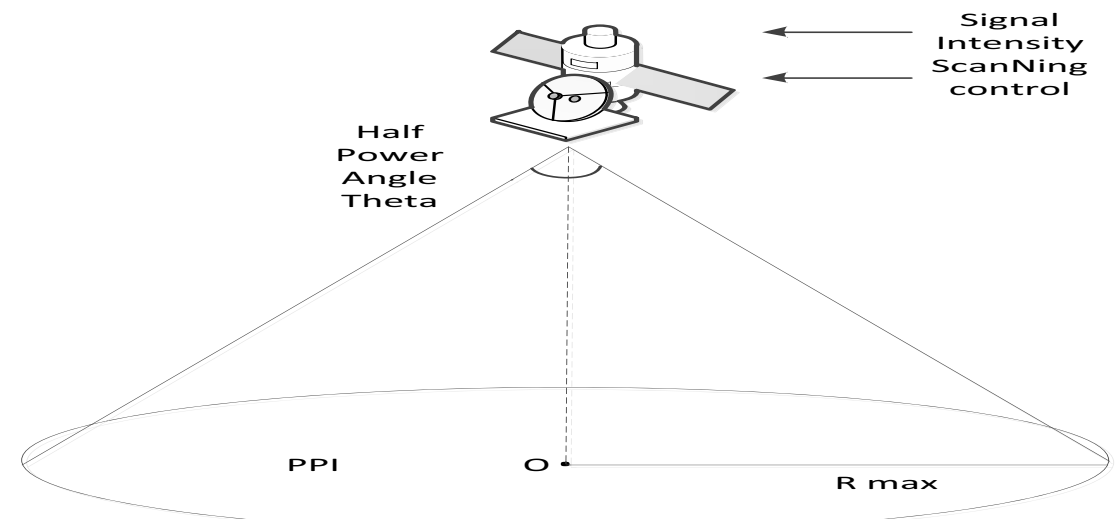

Figure.1 Schematic diagram of single-satellite beam scanning

As shown in Fig. 1, the beam information transmitted by the satellite can form an approximately circular "spot" on the ground [4], and all the receivers in this "spot" can receive the beam information. A circular map-style image of the "spot" and the centre position of the "spot" are displayed on the receiver's flat panel display (PPI). By controlling the centre position of the beam scanning, the satellite can realize the full scan of the scanning area, so that all the targets in the scanning area can be detected. The target can realize the solution of the position coordinates according to the received beam information.

For a certain target area, the position information of the satellite is the main factor to determine the positioning accuracy [5-7]. When the satellite deviates from the target area, the target area is mainly scanned with a smaller half-power angle to improve the accuracy of satellite positioning. When the satellite is moving closer to the target area, the positioning accuracy of the satellite will be improved. In order to meet the requirement of positioning accuracy, we use the neural network BP algorithm.

\section{Neural Network}

Neural network can be divided into biological neural network and artificial neural network. Biological neural network: generally, refers to the biological brain neurons, cells, contacts and other components of the network for the production of biological consciousness, to help biological thinking and action [8]. Artificial Neural Networks (ANNs) are networks of widely parallel interconnected networks composed of adaptive simple units, which are able to simulate the interaction of the biological nervous system with real-world objects. This network relies on the complexity of the system, by adjusting the internal connections among a large number of nodes, so as achieve the purpose of processing information $[9,10]$.

\subsection{Neural Network Introduction}

Neural network is composed of a large number of simple processing units, connected by variable weight of the parallel-distributed system. Neuron is the basic processing unit of artificial neural network; it is a multi-input-single output nonlinear device [11,12]. The structure is showing in Fig.2. In Figure.2, $x_{i}$ is the input signal, $w_{i j}$ is the connection weight from the ith neuron to the jth neuron, $\theta_{j}$ and is the threshold value of the jth neuron. Let $s_{j}$ be an external input signal and $y_{j}$ be an output signal. The transformation of the jth neuron in the above model can be described as: 


$$
y_{i}=f\left(\sum_{j} w_{i j} x_{i}-\theta_{j}+s_{j}\right)
$$

Where the non-linear function $\mathrm{f}(\mathrm{x})$ used here can be a step function, a piecewise function, and a sigmoid type function.

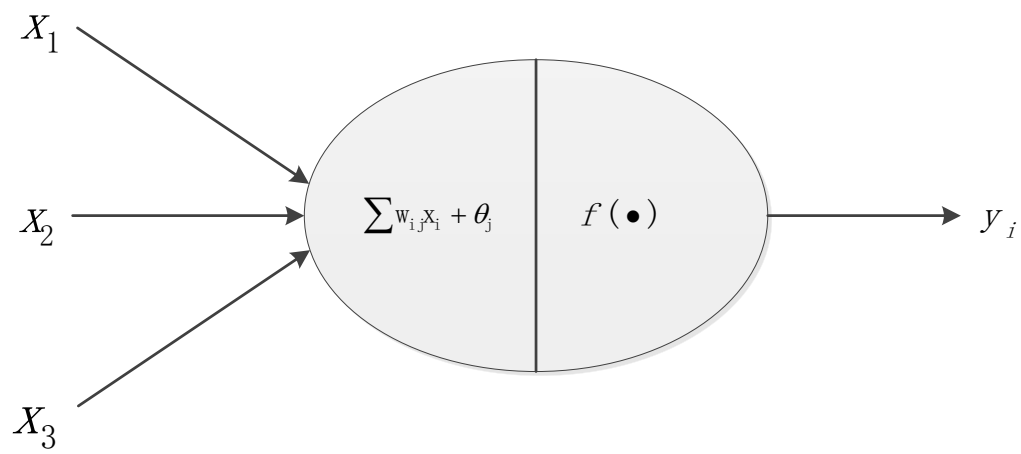

Figure.2 General Description of neuron

For each neuron, there is an output, and through the connection weights to the output to its connected processing unit, the output signal directly depends on the state of the processing unit or stimulus value. This dependency is represented by the effect of the output transform function $f_{j}$ on the processing unit $u_{j}$. Suppose we use $z_{j}(t)$ to define the $u_{i}$ output of the neuron at time $\mathrm{t}$

Or in vector form

$$
z_{j}(t)=f_{j}\left(x_{j}(t)\right)
$$

$$
Z(t)=f(X(t))
$$

Where, $\mathrm{Z}(\mathrm{t})$ is the output vector of the neural network, $\mathrm{f}$ is defining as the corresponding function of the state vector and each component, usually bounded on the interval $(0,1)$.

\subsection{Single Satellite Location Algorithm Based on BP Neural Network}

BP neural network is a multi-layer feed forward neural network, the main characteristics of the network is the signal forward transmission, error back transfer. In the forward transmission, the input signal from the input layer through the hidden layer by layer to the output layer, each layer of the neuron state only affects the next layer of neuron state. If the output layer does not get the desired output, it goes back-propagation and adjusts the network threshold and weight according to the prediction error, so that the BP neural network can predict the expected output continuously. The topological structure of BP neural network is showing in Figure. 3 


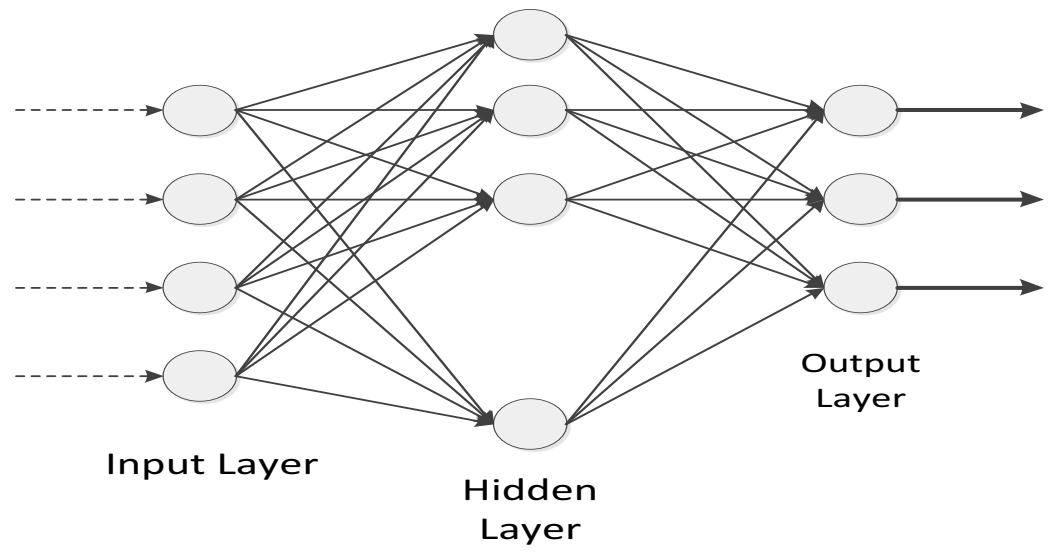

Figure.3 Topological structure of BP neural network

The main idea of the error back propagation algorithm is to divide the learning process into two phases: the first stage (forward propagation process), which gives the input information through the input layer through the hidden layer processing and calculate the actual output value of each unit; (Reverse process). If the desired output value is not obtained at the output layer, the difference (i.e., error) between the actual output and the desired output is recursively calculated on a layer-by-layer basis so as to adjust the weight value according to the difference, it is possible to calculate the product of the error value of the reception unit and the activation value of the transmission unit for each weight. BP neural network based on multi-layer feed forward network structure is showing in Figure.4.

This network not only has input layer nodes, output layer nodes, but also one or more layers of hidden nodes. For the input information, first propagate to the node of the hidden layer, and then propagate the output information of the hidden node to the output node after the activation function (also called the function function, transfer function) of each element. Finally, result. The learning process of the network consists of forward and reverse propagation.

Error inversion (learning algorithm)

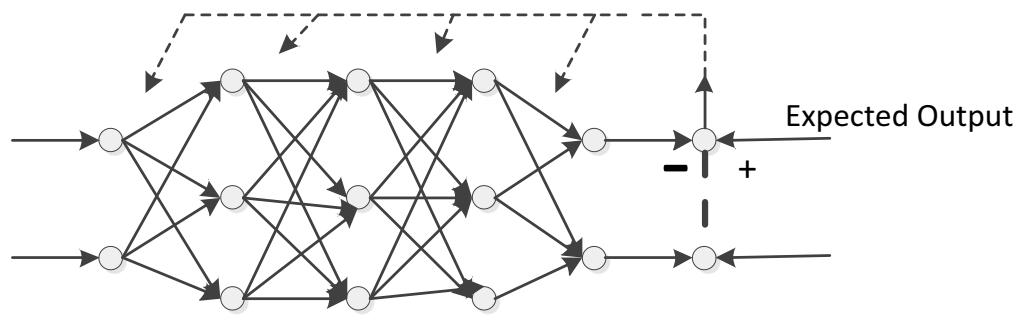

Hidden Layer

Signal Flow

Figure.4 Multi - layer Feedforward Network Structure Based on BP Algorithm

All received beam information is substituting into the neural network to learn and predict the result. $(X, Y)$ is set to be $X=\left[R X_{1}, R X_{2}, \cdots R X_{m}\right]^{T}, Y=\left[r x_{1}, r x_{2}, \cdots, r x_{m}\right]^{T}, R X=\left[x_{1}, x_{2}, \cdots x_{N}\right]^{T}$ is the $\mathrm{X}$ coordinate of all the beam information received by the receiving device at a certain location, and $\mathrm{X}$ is the $\mathrm{X}$ of all the beam information received by the $\mathrm{m}$ experiments. $\mathrm{X}$ is the $\mathrm{X}$ coordinate of the received beam. In addition, $\mathrm{Y}$ is the coordinate of the receiving device in the $\mathrm{X}$ direction. And the hidden layer neuron is $O=\left[O_{1}, O_{2}, \cdots O_{l}\right]$, the weight matrix $W^{1}$ between the input layer and the 
hidden layer neuron, and the weight between the hidden layer and output layer neuron $W^{2}$ respectively.

$$
W^{1}=\left[\begin{array}{cccc}
w_{11}^{1} & w_{12}^{1} & \cdots & w_{1 m}^{1} \\
w_{21}^{1} & w_{22}^{1} & \cdots & w_{2 m}^{1} \\
\vdots & \vdots & \vdots & \vdots \\
w_{l 1}^{1} & w_{l 2}^{1} & \cdots & w_{l m}^{1}
\end{array}\right], W^{2}=\left[\begin{array}{cccc}
w_{11}^{2} & w_{12}^{2} & \cdots & w_{1 l}^{2} \\
w_{21}^{2} & w_{22}^{2} & \cdots & w_{2 l}^{1} \\
\vdots & \vdots & \vdots & \vdots \\
w_{n 1}^{2} & w_{n 2}^{2} & \cdots & w_{n l}^{2}
\end{array}\right]
$$

Where, $\mathrm{m}$ is the number of neurons in the input layer, 1 is the number of hidden layer neurons, $\mathrm{n}$ is the number of output layer neurons. The threshold $\theta^{1}$ of hidden layer neurons and the threshold $\theta^{2}$ of output layer neurons are respectively

$$
\theta^{1}=\left[\theta_{1}^{1}, \theta_{2}^{1}, \cdots \theta_{l}^{1}\right]^{T}, \theta^{2}=\left[\theta_{1}^{2}, \theta_{2}^{2}, \cdots \theta_{n}^{2}\right]^{T}
$$

The output of hidden layer neurons is

$$
o_{j}=f\left(\sum_{i=1}^{m} w_{j i}^{1} x_{i}-\theta_{j}^{1}\right)=f\left(n t_{j}\right), j=1,2, \cdots, l
$$

The output of neurons in the output layer is

$$
z_{k}=g\left(\sum_{j=1}^{l} w_{k j}^{2} o_{j}-\theta_{k}^{2}\right)=g\left(n e t_{k}\right), k=1,2, \cdots, n
$$

Network output and the expected value of the error

$$
\begin{aligned}
& E=\frac{1}{2} \sum_{k=1}^{n}\left(r x_{k}-z_{k}\right)^{2} \\
& =\frac{1}{2} \sum_{k=1}^{n}\left(r x_{k}-g\left(\sum_{j=1}^{l} w_{k j}^{2} o_{j}-\theta_{k}^{2}\right)\right)^{2} \\
& =\frac{1}{2} \sum_{k=1}^{n}\left\{r x_{k}-g\left[\sum_{j=1}^{l} w_{k j}^{2} f\left(\sum_{i=1}^{m} w_{i j}^{1} x_{i}-\theta_{j}^{1}\right)-\theta_{k}^{2}\right]\right\}^{2}
\end{aligned}
$$

The partial derivative of the error $\mathrm{E}$ for the weight $\mathrm{w}_{-} \mathrm{kj}^{\wedge} 2$ between the hidden layer and the output layer neuron is

$$
\frac{\partial E}{\partial w_{k j}^{2}}=\frac{\partial E}{\partial z_{k}} \frac{\partial z_{k}}{\partial w_{k j}^{2}}=-\left(y_{k}-z_{k}\right) g^{\prime}\left(n e t_{k}\right) o_{j}=-\delta_{k}^{2} o_{j}
$$

where, $\delta_{k}^{2}=\left(y_{k}-z_{k}\right) g^{\prime}\left(\right.$ net $\left._{k}\right)$.

The partial derivative of error $\mathrm{E}$ for the weights $w_{j i}^{1}$ between input and hidden neurons is

$$
\frac{\partial E}{\partial w_{j i}^{1}}=\sum_{k=1}^{n} \sum_{j=1}^{l} \frac{\partial E}{\partial z_{k}} \frac{\partial z_{k}}{\partial o_{j}} \frac{\partial o_{j}}{\partial w_{j i}^{1}}=\sum_{k=1}^{n}\left(y_{k}-z_{k}\right) g^{\prime}\left(\text { net }_{k}\right) w_{k j}^{2} f^{\prime}\left(\text { net }_{j}\right) x_{i}=\delta_{j}^{1}
$$

By (9) and (10) available weight adjustment formula for 


$$
\begin{aligned}
& w_{j i}^{1}(t+1)=w_{j i}^{1}(t)+\Delta w_{j i}^{1}=w_{j i}^{1}(t)-\eta^{1} \frac{\partial E}{\partial w_{k j}^{1}}=w_{j i}^{1}(t)+\eta^{1} \delta_{j}^{1} x_{i} \\
& w_{k j}^{2}(t+1)=w_{k j}^{2}(t)+\Delta w_{k j}^{2}=w_{k j}^{2}(t)-\eta^{2} \frac{\partial E}{\partial w_{k j}^{2}}=w_{k j}^{2}(t)+\eta^{2} \delta_{k}^{2} o_{j}
\end{aligned}
$$

Where $\eta^{1}$ and $\eta^{2}$ are the learning steps of the hidden layer and the output layer, respectively.

Similarly, the partial derivative of the error E to the threshold $\theta_{k}^{2}$ of the output layer neuron is

$$
\frac{\partial E}{\partial \theta_{k}^{2}}=\frac{\partial E}{\partial z_{k}} \frac{\partial z_{k}}{\partial \theta_{k}^{2}}=\left(y_{k}-z_{k}\right) g^{`}\left(n e t_{k}\right)=\delta_{k}^{2}
$$

The partial derivative of error $\mathrm{E}$ for the threshold $\theta_{j}^{1}$ of hidden neurons is

$$
\frac{\partial E}{\partial w_{j i}^{1}}=\sum_{k=1}^{n} \sum_{j=1}^{l} \frac{\partial E}{\partial z_{k}} \frac{\partial z_{k}}{\partial o_{j}} \frac{\partial o_{j}}{\partial w_{j i}^{1}}=\sum_{k=1}^{n}\left(y_{k}-z_{k}\right) g^{\prime}\left(n e t_{k}\right) w_{k j}^{2} f^{\prime}\left(n e t_{j}\right) x_{i}=\delta_{j}^{1}
$$

From equation, (12) and (13) can be adjusted threshold for the formula

$$
\begin{aligned}
& \theta_{j}^{1}(t+1)=\theta_{j}^{1}(t)+\Delta \theta_{j}^{1}=\theta_{j}^{1}(t)-\eta^{1} \frac{\partial E}{\partial \theta_{j}^{1}}=\theta_{j}^{1}(t)+\eta^{1} \delta_{j}^{1} \\
& \theta_{k}^{2}(t+1)=\theta_{k}^{2}(t)+\Delta \theta_{k}^{2}=\theta_{k}^{2}(t)-\eta^{2} \frac{\partial E}{\partial \theta_{k}^{2}}=\theta_{k}^{2}(t)+\eta^{2} \delta_{k}^{2}
\end{aligned}
$$

By predicting the output of the neural network, the optimal prediction results are obtaining by constantly modifying the network thresholds and weights.

\section{SIMULATION}

According to our study, the positioning accuracy and the relative position of the satellite with the scanning area are highly correlated. Therefore, we selected the data of seven positioning moments of the apogee, 30 minutes apart for each positioning time point and 3 hours for the selected positioning point. The latitude and longitude corresponding to the position of the satellite at the seven positioning time points are showing in Table 1. The time points of the seven positioning points are T1, T2, T3, T4, T5, T6 and T7.

Table 1 satellite positioning time corresponding latitude and longitude

\begin{tabular}{|c|c|c|c|c|c|c|c|}
\hline $\begin{array}{c}\text { positioning time } \\
\text { lat/lon }\end{array}$ & $\mathrm{T} 1$ & $\mathrm{~T} 2$ & $\mathrm{~T} 3$ & $\mathrm{~T} 4$ & $\mathrm{~T} 5$ & $\mathrm{~T} 6$ & $\mathrm{~T} 7$ \\
\hline latitude & 30.194 & 14.002 & 1.333 & -10.035 & -21.401 & -34.061 & -50.218 \\
\hline longitude & 112.254 & 106.421 & 100.047 & 93.542 & 87.112 & 81.034 & 76.185 \\
\hline
\end{tabular}

We study the position of $\theta=0.5^{\circ}, \mathrm{d}=10 \mathrm{~km}$, and choose the information of 2000 points as the training set of the neural network. We can set the parameter of BP network; predict the output of any 100 coordinates of the location information. The obtained prediction results are showing in Figure.5 and Figure.6. 


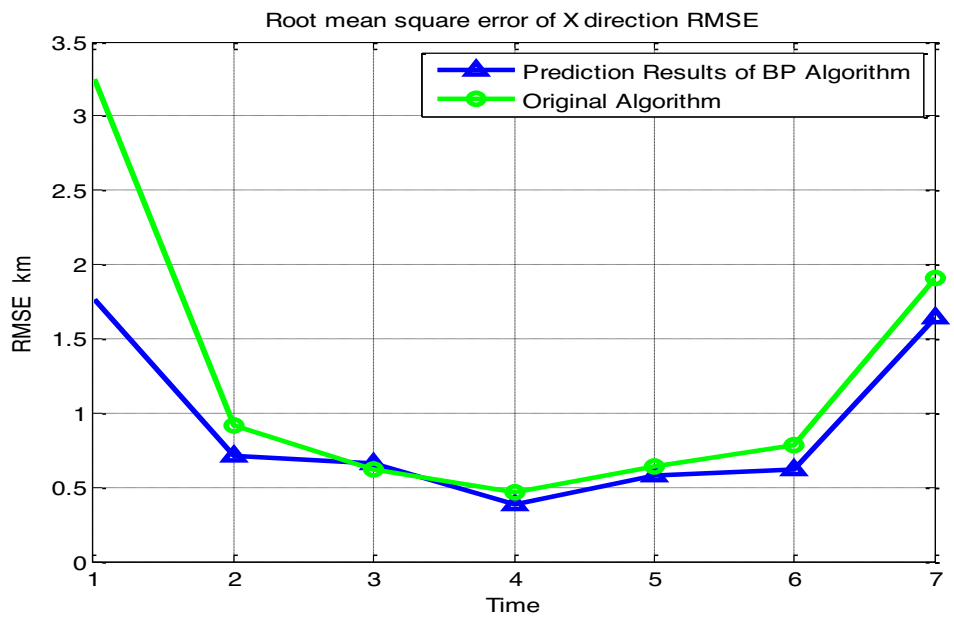

Figure.5 Root-mean-square error of BP prediction in X direction

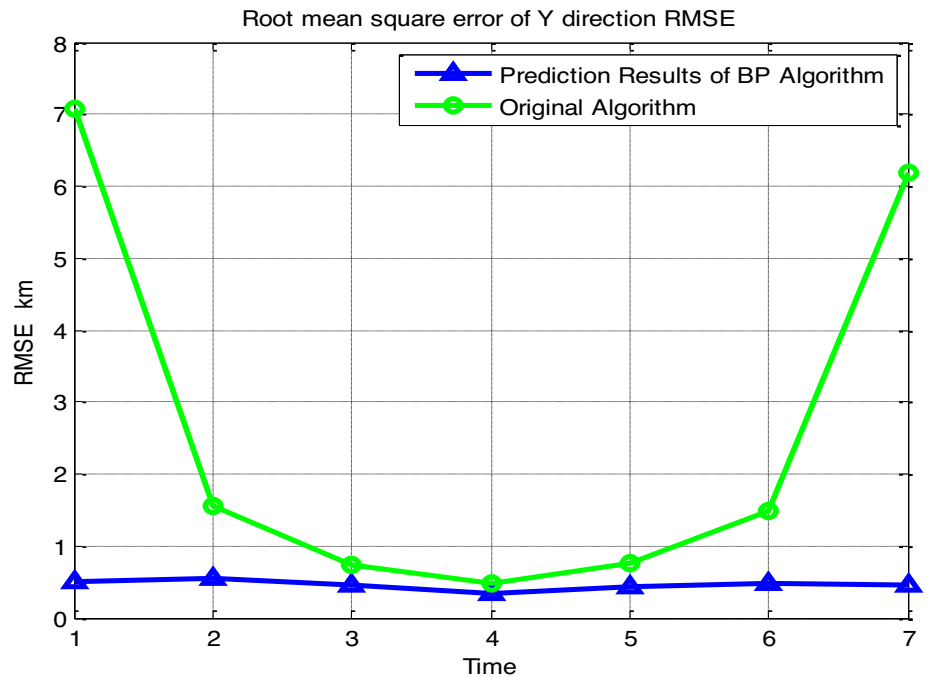

Figure.6 Root-mean-square error of BP prediction in Y direction

From the above output results, BP neural network has a good learning function, can better predict the output of the true location of the target information. Compared with the original algorithm, especially for the satellite deviation from the target area, the accuracy is more obvious the accuracy of BP prediction output is improved. We can see from the result, which BP neural network can improve the positioning accuracy of single-satellite beam scanning.

\section{Conclusion}

This paper, the neural network BP algorithm combined with beam scanning is using to calculate the position coordinates of the receiver. The beam scanning positioning technique is used to detect the target and to calculate the coordinates of the target position. When a satellite is in the far near the site, the satellite can cover the target area for a long time, the receiver within the target area in the period of the satellite coverage within the received beam scanning information of the satellite receiver to collect enough information through the beam signal processing, positioning calculation can calculate their 
longitude coordinate degree. In this paper, we proposed a new method: neural network combined with beam scanning, it is based on the scanning beam received to calculate the location information.

\section{References}

1. Bagrov A V, Leonov V A, Mitkin A S, et al. Single-satellite global positioning system [J]. Acta Astronautica, 2015, 117:332-337.

2. Zhang J, Lv J, Yu Y. Research on the Positioning by Single Satellite in Constellation Communication Systems [J]. Journal of Telemetry Tracking \& Command, 2009.

3. Zhao M, Wang Y, Du S, et al. The study of rapid localization algorithm from beam scanning by single satellite based on neural network[C]// IEEE International Conference on Computer Communication \& the Internet. IEEE, 2016:186-190.

4. Harchowdhury A, Sarkar B K, Bandyopadhyay K K. Beam scanning for reception performance improvement of satellite-based AIS[C]// IEEE International Conference on Aerospace Electronics and Remote Sensing Technology. IEEE, 2015:1-5.

5. An-Nan L U, Kong X Z. Passive localization from frequency measurements by single satellite [J]. Journal of China Institute of Communications, 2004.

6. Lu A N, Yang X N. Passive location from the combined set of frequency and phase difference measurements by single satellite [J]. Systems Engineering \& Electronics, 2010, 32(2):244-247.

7. Chan Y T, Towers J J. Passive localization from Doppler shifted frequency measurements[C]// International Conference on Acoustics, Speech, and Signal Processing. IEEE, 1991:1465-1468 vol.2.

8. Wang Y. GENERALIZED NEURAL NETWORK SYSTEM AND BP ALGORITHM[J]. Minimicro Systems, 2001.

9. Liang J Z, He X G, Zhou J Q. DYNAMIC ANALYSIS OF BP ALGORITHM FOR NEURAL NETWORKS[J]. 2002(5):729-735.

10. Zhang H. A New BP Algorithm of Multiplayer Neural Network[J]. Communications Technology, 2003.

11. Jing G, Du W, Guo Y. Studies on prediction of separation percent in electrodialysis process via BP neural networks and improved BP algorithms[J]. Desalination, 2012, 291(14):78-93.

12. Yu F, Xu X. A short-term load forecasting model of natural gas based on optimized genetic algorithm and improved BP neural network[J]. Applied Energy, 2014, 134(134):102-113. 